\title{
Erratum to: Impact of oral hygiene involving toothbrushing versus chlorhexidine in the prevention of ventilator-associated pneumonia: a randomized study
}

Claudia Fernanda de Lacerda Vidal ${ }^{1 *}$, Aurora Karla de Lacerda Vidal ${ }^{2}$, José Gildo de Moura Monteiro Jr. ${ }^{3}$, Aracele Cavalcanti ${ }^{4}$, Ana Paula da Costa Henriques ${ }^{5}$, Márcia Oliveira ${ }^{6}$, Michele Godoy ${ }^{7}$, Mirella Coutinho ${ }^{7}$, Pollyanna Dutra Sobral ${ }^{7}$, Claudia Ângela Vilela ${ }^{7}$, Bárbara Gomes ${ }^{7}$, Marta Amorim Leandro ${ }^{8}$, Ulisses Montarroyos ${ }^{9}$, Ricardo de Alencar Ximenes ${ }^{10}$ and Heloísa Ramos Lacerda ${ }^{11}$

\section{Erratum}

In the version of this article that was originally published [1] there was an error with the author name "Ana Paula da Costa Henriques" since it was incorrectly listed as "Ana Paula Trindade Henriques". The original article has been revised.
Received: 20 February 2017 Accepted: 20 February 2017

Published online: 27 February 2017

\section{Reference}

1. De Lacerda Vidal CF, De Lacerda Vidal AK, de Moura Monteriro JG, Cavalcanti A, da Costa Henriques AP, et al. Impact of oral hygiene involving toothbrushing versus chlorhexidine in the prevention of ventilatorassociated pneumonia: a randomized study. BMC Infect Dis. 2017;17:112.

\begin{abstract}
Author details
${ }^{1}$ Tropical Medicine Health Sciences Center, Committee on Infection Control of Hospital das Clinicas, Universidade Federal de Pernambuco, Av. Professor Moraes Rego, 1235 Hospital das Clínicas - Cidade Universitária, Recife, Pernambuco 50670-901, Brazil. ²Department of Pathology, Institute of Biological Sciences, Universidade de Pernambuco, Hospital de Câncer de Pernambuco, Real Hospital Português de Beneficência em Pernambuco, Recife, Pernambuco, Brazil. ${ }^{3}$ Cardiac Intensive Care Unit, Pronto-Socorro Cardiológico de Pernambuco, Universidade de Pernambuco, Recife, Pernambuco, Brazil. ${ }^{4}$ Committee on Infection Control, Pronto-Socorro Cardiológico de Pernambuco, Universidade de Pernambuco, Recife, Pernambuco, Brazil. ${ }^{5}$ Committee on Infection Control, Real Hospital Português de Beneficência em Pernambuco, Recife, Pernambuco, Brazil. ${ }^{6}$ Intensive Care Unit, Hospital Agamenon Magalhães, Secretaria de Saúde de Pernambuco, Recife, Pernambuco, Brazil. 'Intensive Care Unit, Hospital das Clínicas, Universidade Federal de Pernambuco, Recife, Pernambuco, Brazil. ${ }^{8}$ Committee on Infection Control of Hospital das Clinicas, Universidade Federal de Pernambuco, Recife, Pernambuco, Brazil. ${ }^{9}$ Institute of Biological Sciences, Universidade de Pernambuco, Recife, Pernambuco, Brazil. ${ }^{10}$ Faculty of Medical Sciences, Tropical Medicine Health Sciences Center, Universidade Federal de Pernambuco, Recife, Pernambuco, Brazil. ${ }^{11}$ Department of Infectious and Parasitic Diseases, Faculty of Medical Sciences, Tropical Medicine Health Sciences Center, Recife, Pernambuco, Brazil.
\end{abstract}

\footnotetext{
* Correspondence: vidal.claudia@gmail.com

${ }^{1}$ Tropical Medicine Health Sciences Center, Committee on Infection Control of Hospital das Clinicas, Universidade Federal de Pernambuco, Av. Professor Moraes Rego, 1235 Hospital das Clínicas - Cidade Universitária, Recife, Pernambuco 50670-901, Brazil
} the Creative Commons license, and indicate if changes were made. The Creative Commons Public Domain Dedication waiver (http://creativecommons.org/publicdomain/zero/1.0/) applies to the data made available in this article, unless otherwise stated. 